\title{
Hypertension-Your Foods and Your Body
}

\section{Bush T*}

Bioindivdual Nutrition, Nutrimom ${ }^{\circledast}$ Inc. Pfafftown, North Carolina, USA

*Corresponding author: Bush T, Founder/President, Bioindivdual Nutrition, Nutrimom ${ }^{\circledR}$ Inc. Pfafftown, North Carolina, USA, Tel: 3364861905; Email: Nutrimom@yahoo.com

Received date: March 02, 2017; Accepted date: March 13, 2017; Published date: March 28, 2017

Citation: Bush T. Hypertension-Your Foods and Your Body. 2017. 3:1.

Copyright: (c) 2017 Bush T. This is an open-access article distributed under the terms of the Creative Commons Attribution License, which permits unrestricted use, distribution, and reproduction in any medium, provided the original author and source are credited.

\section{Introduction}

The way the body reacts to different factors can sometimes be difficult to diagnose correctly. In a world where hypertension is often treated as the main symptom, patients are increasingly searching to dig deeper on the true cause versus standardized treatment. Patients are becoming more in touch with their bodies including how the original ailments began. With treatment alternatives expanding and medical professionals beginning to see the benefits of a broader range of whole health treatment plans for their patients, the medical world is being taken by storm. Foods are now one of the top sources of a patient's health examination whether seeming immediately relevant or not.

\section{Hypertension's Source}

For most adults, there's no unusual cause of high blood pressure other than the typical sources. With healthcare systems limiting time with patient visits, underlying causes can easily be disregarded. Often the response is "This type of high blood pressure, called primary (essential) hypertension, tends to develop gradually over many years"[1] leaving many with a feeling of inadequacy in their personal goals in regards to lack of proper exercise, foods that they may overindulge in or accepting that age is to blame. However, integrative and alternative physicians are now broadening the health spectrum to include essential dynamics. "Some people have high blood pressure caused by an underlying condition. This type of high blood pressure, called Secondary Hypertension, tends to appear suddenly and cause higher blood pressure than does primary hypertension" [1]. One such way is to look at what causes our metabolism to react.

Our body is made up of specific hormones; a delicate balance that sustains how our blood sugar levels work how our heart pumps our blood and how our nervous system works. It is extremely obvious to understand that one imbalance can cause a chain reaction causing a domino effect within our body. One of the key hormones is cortisol. Although this hormone is in place and its purpose is to aid our body's normal responses, a hiccup in this hormone can also begin a silent cycle of a multitude of system breakdowns. Known as the stress hormone, a body that is always in high cortisol mode means a body that is in constant flight or fight mode. "Over time, repeated activation of the stress response takes a toll on the body. Research suggests that chronic stress contributes to high blood pressure, promotes the formation of artery-clogging deposits, and causes brain changes that may contribute to anxiety, depression, and addiction" [2]. This is when your body begins to break down.

\section{The Crossover}

Once the body begins to react, a chain of events begins. A study shared on NCBI suggests that effected cortisol levels can play a role in allergies and allergic symptoms. The association between salivary cortisol levels in infancy and allergic sensitization and allergic symptoms suggests a role of an altered hypothalamic-pituitary-adrenal axis in the etiological process of allergies [3]. Take one piece of the puzzle and try to fit in the wrong piece and you are left with something this is incomplete. Our bodies must be complete to do what they need to do on a daily basis. Continuous elevated cortisol levels can begin to eat their way into other areas of our health, including having our bodies become hypersensitive. When you are stressed, you are put on the defence; the same happens to your body. These changes help us survive short periods of stress, but they hurt us when they continue for years [4].

While cortisol is one of the key places to look for an underlying health issue, we also need to look at the places that are directly affected by the malfunction. Our stomach houses more neurons than our entire spine. Neurons are the messengers that ensure our body gets all of the correct signs of what to do and how to act. Undiagnosed food allergies and intolerances are an easy highway to setting off the wrong signals. If your gut is not optimal, neither are the rest of you. It is very possible that the foods that you are eating are the cause of your hypertension as well as other ailments. This may not be the case for everyone but it is a theory that can be tested without the use of medications or the possibility of side effects from medications given as a symptom band aid.

\section{Hypertension Check-up}

Rather than treating one symptom treat the patient with a whole health plan. Rather than give medications or prescriptions that may add additional symptoms, first exhaust all other 
methods. This is itself also enables each patient to feel like a person treated as a person and not just a number fit into a time slot [5].

\section{Check for more than just the typical array of blood work}

The worst thing to tell a patient who is unwell is that all of their blood work is "within normal range" when, in fact, it's only based upon the range of blood work that you are willing to check. Check everything, even if you don't normally agree with that type of testing.

\section{Test for hormones, food allergies and sensitivities}

Without having everything, you are only working on a small piece of your patient's symptom and not the cause. All of these can be treated together and on an individual's specific level vs. prescribed generic written prescriptions.

\section{Understand that you may not be able to help as much as a patient needs it}

If you are part of the group of physicians that doesn't necessarily agree with out-of-the-box thinking, then at least be willing to begin the journey with your patient but also be professional enough to let them know you may not be the best resource for them to finish with. Not every doctor specializes in every health aspect; be a better person to yourself and your patients and refers them to those who can help them.

\section{Do research}

No eye-rolling, just roll up your sleeves and try it. You may not agree, understand or accept it even after you have researched these newer methods but finding the cause of hypertension and other health issues is never about you; it is always about the patient.

Once adequate testing is complete, present multiple treatment options to your patient. Hypertension caused by high cortisol can be trailed safely with the appropriate safe foods as well as methods such as meditation, acupuncture, taking time to be outside with nature, etc. I recommend finding the right foods first as specific foods have proven to lower hypertension and will also begin the healing process in the gut to, in turn; begin to heal the rest of the body. "Your adrenal glands are two almondsize glands that sit on top of the kidneys. The adrenal glands produce a variety of hormones, including cortisol (an antiinflammatory hormone) which normally serves to prevent allergic reactions. Normally, when one is exposed to an irritating substance, the body increases its production of these antiinflammatory hormones which prevents an allergic response from occurring. Most individuals suffer from allergies because they are not producing adequate amounts of adrenal gland hormones. Often the problem is chronic and worsens when the person is fatigued, or under stress" [6].

By eliminating and replacing offending foods (either IgG or IgG) the patient can begin to treat the cause of their hypertension. Very specific nutritional help should be offered and I strongly recommend a person that specializes in foods beyond just the Nutrition or Dietician aspect. Patients working with a Nutritionist or Dietician may receive some helpful food information but it will be loosely based on fat, calorie, etc. content rather than foods that are or are not allowed for the person they are counselling. They may give generic meal plans and they may or may not offer support to a certain extent but having a wide range of food allergy and intolerance knowledge is the key.

The term for non-prescription methods that has been paired with this or similar treatments is known as Nutraceuticals. "Various terms have been used interchangeably to designate foods for disease prevention and health promotion. The term Nutraceuticals was introduced in 1989 by the US Foundation for Innovation in Medicine and referred to "any substance that is a food or a part of a food and provides medical or health benefits, including the prevention and treatment of disease" [7]. Through research, we have found that other countries and cultures have used this methodology for centuries with a proven track record of lower cardiovascular issues as well as food allergies. To know which foods are uniquely aggravating your body means a beginning step to not only improved cardiovascular health but other areas of health in the process. "When a new testing for hidden food allergens came out a few years ago she was one of the first people that I tested. Surprisingly for both of us she came back highly allergic to eggs. This is not the regular kind of allergy that would show itself with a rash or another physical symptom right away. This was a hidden food allergy that can be present without any obvious, physical symptoms that you would see from the outside. Hidden allergies last 2 to 20 days. So she removed all eggs from her diet." Two weeks later we tested my mom's blood pressure and it was completely normal for the first time in 20 years. With the consent and help of her wonderful medical doctor we decreased that amount of medication slowly and within a month she was completely drug free with a normal blood pressure. She continues well to this day" [8]. If the proof is apparent and the patient is happy, are we not doing what we were meant to do?

\section{An Example for the Case Study}

A female client that I consulted in her late thirties presented with complaints of fatigue, weight gain, extreme anxiety and depression, among other ailments. Her medical records indicated elevated levels of blood pressure as well as cortisol, low thyroid and she also tested positive for Candida (yeast overgrowth). "Candida seems to be at the foundation of almost everyone's poor health and with good reason" [9]. She was irritable and admitted that she was neither a person who liked to cook nor did she like the majority of the foods that I recommended. After much additional check-in, some advice on how to utilize the correct foods in ways that were more convenient to her lifestyle and a bit of patience, she began her journey. Months later, she came to me at a local event. She was glowing and lost over thirty pounds. Her reason for the visit "I just came here to thank you for helping me, for making me feel like an individual and not another copy and paste document. I feel amazing for the first time in many, many years." 


\section{Conclusion}

Within the years that I have been referred client consultations, I have seen how foods can improve various health issues. Is food the answer to everything? No but it's the place that is commonly overlooked and yet the easiest place to begin.

\section{References}

1. Mayo Clinic (2016) Basic causes: High blood pressure.

2. Harvard Health (2011) Understanding the stress response. A Harvard health Publication.

3. Stenius F, Borres M, Bottai M, Lilja G, Lindblad F, et al. (2011) Salivary cortisol levels and allergy in children: The Aladdin birth cohort. J Allergy Clin Immunol 128: 1335-1339.
4. Whiticomb J (2011) Reducing the risks of high cortisol. Life Extension Magazine.

5. Grisanti R (2017) Leaky Gut: Can this be destroying your health? Functional Medical University, USA.

6. Analytical Research Labs, Inc. (1987) Nutritional causes of allergies.

7. Alissa EM, Ferns GA (2012) Functional foods and nutraceuticals in the primary prevention of cardiovascular diseases. J Nutr Metabol 2012: 569486

8. Nenninger S (2013) High blood pressure and hidden food allergies. Natural Medicine.

9. Edwards M (2014) Hypertension-How to lower your blood pressure quickly and naturally? Organic Lifestyle. 